\title{
A ROBOTIC SYSTEM FOR AUTOMATED MASONRY
}

\author{
E. Gambao, C. Balaguer ${ }^{*}$, and F. Gebhart** \\ DISAM. Universidad Politecnica de Madrid. C/Jose Gutierrez Abascal,2. \\ E-28006, Madrid, Spain. \\ gambao@disam.upm.es. Tel +34913363061. Fax +34915642961 \\ *Universidad Carlos III of Madrid, C/Butarque, 15, E-28911 Leganés (Madrid), Spain \\ balaguer@ing.uc3m.es, Tel+34916249426. Fax+34916249430
}

$$
\text { ** LISSMAC, GmbH, Alte Strasse, 12, D-88410 Bad Wurzach, Germany }
$$

\begin{abstract}
The traditional methods for masonry have reached their system limits and cannot contribute to further effectiveness. Further rationalisation can only be achieved by the reduction of labour and construction times. Therefore a certain innovative leap is required by a system approach that combines the already existing construction technologies with new information and robot technologies. An integrated automation system have been developed in the European ESPRIT III ROCCO (Robot Assembly System for Computer Integrated Construction) project. Block assembly tasks are performed by means of two robotics systems. Industrial partners and research centres from Germany, Spain and Belgium that are linked to the construction industry and robotics have formed the consortium. The most significant aspects related to the development of these robotic systems are presented in this paper.
\end{abstract}

Keywords: Computer Integrated Construction, Robotized masonry, Large Range Robots, Adaptive control.

\section{INTRODUCTION}

The conventional masonry process requires highly skilled workmen in order to achieve sufficient and consistent walling quality. This labour intensive construction process results in relatively high costs. To achieve higher rationalisation and humanisation, different methods have been tested [1].

One of the approaches, which is currently used by the sand-lime brick and cellular concrete industry in Europe is the enlargement of the size of the building blocks. The enlarged dimensions of these blocks, however, coincide with their increased physical weight of up to $300 \mathrm{~kg}$. Due to their larger dimensions and heavier weights these building blocks are not ergonomically desirable and, therefore, various mechanical aid-devices such as hydraulic balancers or minicranes with counterweights are used for the assembly.

Another tendency, which is steadily gaining momentum is the use of exact, plane-parallel blocks, which are laid on a thin mortar laid, respective glue bed, or assembled dry and filled with concrete after the assembly of the whole wall like shuttering blocks. These methods also offer better physical properties like heat insulation and bigger load bearing capacity because of the lack of mortar joints. Because of the reduction of the walling operations they also enable higher working speed and easier use. A first layer of smaller blocks in a more or less thick mortar bed usually compensates the different accuracies of the floor. By applying this method the difficult alignment, which is required for adjusting the position of each block can be reduced. Though an increment of the working speed can be achieved even by employing less qualified workers on the construction site, the present and future share of costs due to labour will be constantly increasing.

Other existing approaches include some degree of automation. Certain activities, such as the cut of the blocks, can be transferred from the construction site to the building material factory. Pre-cutting of blocks simplifies the work avoiding transporting the bigger cutting equipment. However, this method leads to mark the blocks and deliver them together with an assembly plan, which shows the positions of the respective blocks in the wall. So, information technology for the creation of assembly plans and for the control of the cutting equipment is necessary.

Further rationalisation can only be achieved by the reduction of labour and construction times. Since the above mentioned mechanised methods for masonry have reached their limits, they cannot 
contribute to further effectiveness and therefore a certain innovative leap is required by a system approach that combines the already existing construction technologies with new information and robot technologies. This has been the aim of the European ESPRIT III ROCCO (Robot Assembly System for Computer Integrated Construction) project, which deals with the development and realisation of an integrated automation system.

The system approach means for a constructionoriented development of the existing technologies in order to close the gaps between construction technologies and intelligent interfaces. The object is then to provide the necessary flexibility for a one-ofa-kind building production and for a robust design and user friendly programming. To achieve the requirements, the above mentioned basic strategies for automated construction are applied: the information integration, the transfer to the prefabrication (only where applicable) and the redesign of construction materials.

Two different mobile robot systems for the masonry on-site have been developed in the project. The large range of action can be achieved by different robot configurations such as automatic cranes, big SCARA robots, big articulated robots, gantry robots, etc. However, these big and heavy robots are difficult to transport to the construction site, have some unsolved scientific and technical problems, and need a very high investment. An articulated robot placed over a mobile platform (a lorry, a towable platform or an autonomous mobile robot) results very appropriate for the assembly tasks on a construction site. These mobile systems must be able to move inside the building, which can limits their weight and their size. The ROCCO systems are based on articulated robots placed over mobile platforms.

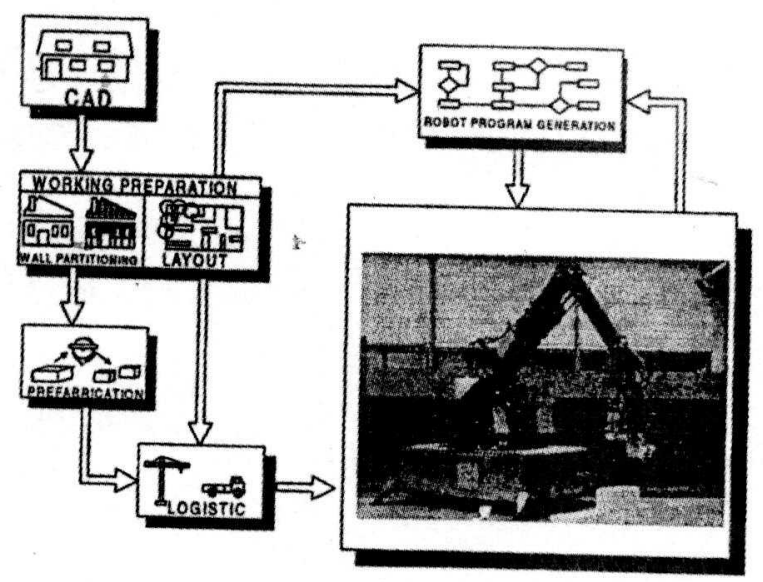

\section{COMPUTER INTEGRATED CONSTRUCTION}

The main goal of the system is the automation of the construction process. Automation and robotics should be taken as part of CIC (Computer Integrated work, through the use of computers and other Construction) concept that tries to integrate each phase of construction operation, from architectural planning $(\mathrm{CAD})$ to the execution of construction communication technologies [3]. The developed CIC concept includes the integration of the following steps (Fig. 1):

- Assisted drawing of the complete building using conventional $\mathrm{CAD}$ system with specially developed tools and interfaces.

- Working planning development which includes the wall partitioning in the elementary parts to be used through the expert knowledge rules, and on site work layout planning.

- Generation of the manufacturing commands for the prefabrication of the elementary parts (blocks). These commands include the control of the manufacturing robots and equipment.

- Development of the logistics of the on site resources supply (parts, material, machines, operators, etc.). The elementary parts (prefabricated and pre-cut blocks) are optimally situated in the on site pallets in order to improve the construction process. In the same way, the optimal position and orientation of pallets and robot on the floor are calculated.

- Robot programs generation, which includes an off-line program for planning of complex assembly tasks and for robot actions generation. The wall assembly system is orginised on the base of the successive generation of the different type of actions.

The robot system is supervised by a friendly man-machine interface (MMI) which permits the graphical simulation of the robot tasks before the real execution. For this simulation the TOROS (Toolbox for Robot Simulation) developed at DISAM is used [4].

\section{THE ROBOTIC SYSTEMS}

After the marketability analysis in Europe and in particular in Germany and Spain, the efforts have been driven towards two different areas:

- Residential building with typical wall height between 2.5 and $3.5 \mathrm{~m}$. The process 
characteristics are very variable respecting distribution and shape, making difficult their automation.

- Industrials building with typical wall height between 8 and $10 \mathrm{~m}$ and with more standardised layout and shape.

The dimensions and features of the robots are according to the typical tasks of the construction industry. The first one, for residential buildings, has a reach of $4.5 \mathrm{~m}$ and it is able to handle up to $400 \mathrm{~kg}$ [5]. This robot is placed over an autonomous vehicle that allows the movement on the construction site (see Figure 1). Its main task is the erection of walls in residential buildings. The second one is able to handle up to $500 \mathrm{~kg}$ with a reach of $8.5 \mathrm{~m}$. This robot is placed over a towable platform and its main task is the erection of external walls in industrial buildings (Figure 2).

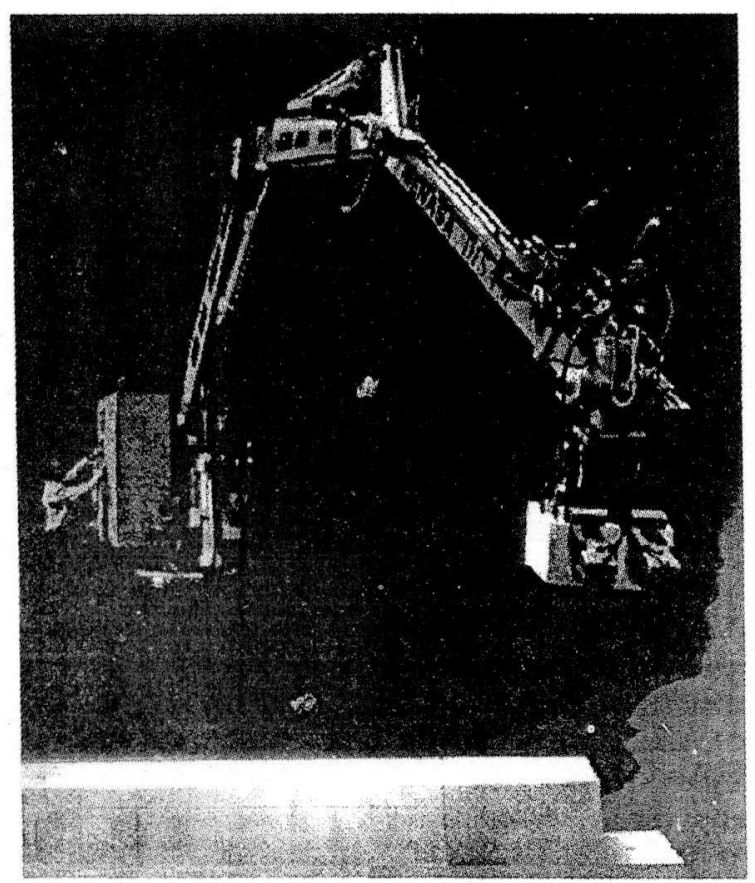

Fig 2.8.5 m ROCCO Robot

\section{A ROBOT CONTROL SYSTEM}

ivany of the service applications such us construction, mining, space or nuclear maintenance, require articulated robots with special features such as large range, large payload capacity, teleoperation facilities, etc., especially for outdoon operations like the presented for the ROCCO systems. Hydraulic actuators results very appropriate for these large robots because their high weight- power ratio. The developments of sophisticated servovalves that allow precise oil flow control have contributed to improve the performance of hydraulic systems. However, several tough problems still remain for the control of such kind of large articulated manipulators hydraulically driven. The static and dynamic system behaviour of these large robots that have low natural frequencies is extremely nonlinear because of both the robot mechanics and the hydraulic actuators [6]. An additional source of difficulty for the control design is the elasticity of the superimposed by the elasticity of the hydraulic fluid within the actuators. As a result of this fact structural vibrations and deflections appear. Moreover, because of the large payload the robot model changes considerably during the work. For all these reasons, conventional control strategies based on constant gain PD/PID controllers with linear models, which usually consider the arm joints in an independent way, results completely ineffective for these robots and more sophisticated control strategies are required [7].

A suitable control strategy has been developed and successfully implemented in the robots. The selected control architecture implements a control strategy based on a gain-scheduling scheme where the controller gains are adjust on the fly be means of a fuzzy supervisory system. The control architecture follows a hierarchical, distributed, non-decoupled control strategy. The control strategy is nondecoupled although each axis has its own controller, because the control parameter computation is performed taking into account all the robot axes [8].

The system dynamic behaviour is well represented by the axes position and the payload. These two variables arc taking into account for the control parameters computation. A fuzzy inference engine is in charge of this computation in real time.

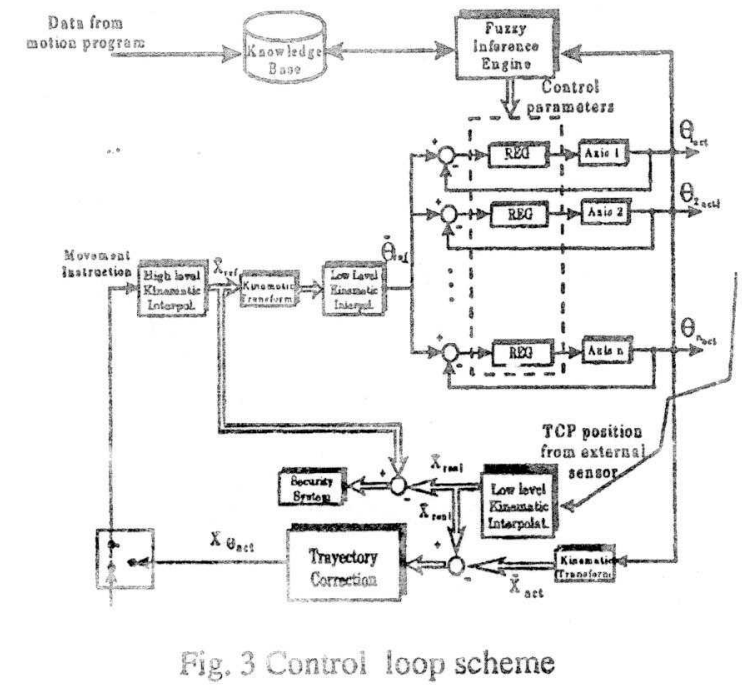


For the industrial building robot, a static deflection compensation system, based on the use of an external position sensor with autotracking, has been implemented. The global external position sensor is used to correct deviations of the robot TCP due to static deflections of the structure. When the precision of the movement must be high, e.g. for picking of placing a block, the actual robot TCP position, measured with the global position system, and the theoretical robot TCP position, computed from the measure from the sensors collocated in each axis (where the static deflections are not included), are compared. If necessary, a correction trajectory is automatically generated as a movement command.

Figure 3 shows the control scheme, including the module for correction of deviations due to static deflections of the robot structure.

A specially developed gripper that includes a passive compliance system has been developed in order to achieve an accuracy that has been set over $\pm 5 \mathrm{~mm}$.

\section{RESULTS}

The obtained systems improve the manual block assembly tasks reducing dramatically the construction time and efforts. The cycle time (pick \& place operation of a $350 \mathrm{~kg}$ block) has been set over $20 \mathrm{sec}$. A single operator is able to manage the complete system by means of an appropriate and friendly interface. Especially attention has been paid to the security of the system and automatic error recovering. The systems allow introducing automatic procedures in an industry that is characterised by traditional and manual methods with hard labour conditions and low productivity. Nowadays the system is been introduced in the market.

\section{ACKNOWLEDGEMENTS}

This work has been developed with the financial support of the European Union into the ESPRIT program frame (ESPRIT $n^{\circ}$ 6450) and with financial help from the CICYT (Spanish Government). The authors wish to thanks the contribution of all the partners in the ROCCO project.

\section{REFERENCES}

[1] F. Gebhart et al., Final Report of the ROCCO project, ESPRIT III Program of the European Union, 1998.

[2] E. Gambao, Hydraulic Robot Control. Application to Large Range Robots for the Construction Industry, Ph. D. Thesis, Polytechnic University of Madrid, 1996.

[3] C. Balaguer, et al., Site Assembly in Construction Industry by means of a Large Range Advanced Robot, $13^{\text {th }}$ ISARC, Tokyo (Japan), 1996.

[4] C. Balaguer, et al., TOROS: Graphical Toolbox for Robot Simulation, International Workshop on Graphics and Robotics, Dagstuhl (Germany), 1993.

[5] D. Spath, et al., Robot System for Residential Building Construction: Integration and Operation, $14^{\text {th }}$ ISARC, Pittsburgh (USA), 1997.

[6] H.B. Kunze, et al., On the Dynamic Control of a Hydraulic Large Range Robot for Construction Applications. Automation in Construction, vol. 4, 1995.

[7] E. Gambao, et al., Robot Assembly System for the Construction Process Automation, 1997 IEEE Int. Conference on Robotics and Automation, ICRA' 97. Albuquerque, New Mexico, (USA), 1997

[8] E. Gambao, et al., Hierarchical Control Architecture for Large Range Robots with Static Deflection Correction. Symposium on Robot Control SYROCO'97. Nantes. France, 1997 